\title{
Tendencias a largo plazo en la financiación de los cuidados a la infancia y a la población dependiente en Europa ${ }^{1}$
}

\section{Paula Rodríguez-Modroño \\ Departamento de Economía Universidad Pablo de Olavide prodmod@upo.es}

\section{Mauricio Matus-López \\ Departamento de Economía Universidad Pablo de Olavide mmatlop@upo.es}

En este artículo se proyecta en un horizonte de cuarenta años el gasto en infancia y el de mayores dependientes para 28 países europeos y se estima su impacto en proporción al cambio demográfico. Para la proyección se utilizan datos de gasto público en infancia y dependencia, crecimiento poblacional y crecimiento del PIB de las bases de datos de Eurostat y del Fondo Monetario Internacional. Con este ejercicio de simulación se identifican los países europeos que, de no cambiar sus políticas, desprotegerán a menores y mayores, 0 a una población más que a otra. Estos resultados permiten anticipar los impactos que la tendencia actual y su sostenimiento tendrán en el futuro y señalar aquellos países que deben revisar su actual diseño de políticas de cuidados a la infancia y a la población dependiente.

\section{Palabras clave}

Políticas familiares, dependencia, gasto social, austeridad.
Familia-politikak, mendekotasuna, gastu soziala, austeritatea. 


\section{Introducción}

Existen dos fuerzas que están modificando los recursos que los Estados de Bienestar europeos dedican a los cuidados a la infancia y a la población mayor dependiente. La primera es el propio cambio demográfico, que ha disminuido la proporción de menores y ampliado la población de mayores. Según los datos de población de Eurostat (2017a), hace veinticinco años había la mitad de menores de 5 años que mayores de 65 en la Unión Europea de los Veintisiete (29,3 y 64,6 millones en 1990, respectivamente). En la actualidad, los menores representan solo un tercio de la población mayor (25,9 y 95,2 millones, respectivamente, en 2015), mientras que en 2060 la población mayor de 65 años en la Unión Europea de los Veintiocho será casi cinco veces la población menor de 6 años.

La segunda fuerza es la contención del gasto público, que se impone con la entrada de los paradigmas neoliberales a comienzos de los años noventa y que se consolida con las políticas de austeridad de los últimos años. Aunque existe un amplio consenso sobre el inicio, hace al menos tres décadas, de un proceso de reconfiguración del Estado de Bienestar en la mayoría de las democracias capitalistas (Taylor-Gooby, 2002; Palier, 2010; Hemerijck, 2012), la recesión de 2008 ha marcado el comienzo de una nueva era de "austeridad permanente" (Pierson, 2002) o, como denomina Jessop (2015), "duradera" o "sostenida". La introducción de duras políticas de austeridad en toda Europa es prueba del empeoramiento de esta tendencia general (Farnsworth e Irving, 2011, 2015; Matsaganis, 2012; Karger, 2014; Pavolini et al., 2015). Estos programas de austeridad están siendo acompañados de recortes sociales, generalmente legitimados con el pretexto de salvar las finanzas públicas y de que la antigua protección social ya no es sostenible en las circunstancias actuales (Romano y Punziano, 2015). Aunque la austeridad se está aplicando en todos los países avanzados, en los países europeos rescatados, sometidos a estrictos programas de reformas, la presión fiscal es aún mayor.

Como consecuencia de estas políticas de recalibración (Ferrera et al., 2000) y de recortes, se está asistiendo al desarrollo de un nuevo contrato social en los países occidentales sobre la base de una nueva combinación o mix de obligaciones familiares y provisión mercantil y pública de los trabajos de cuidados. Respecto a las políticas de atención a la dependencia o cuidados de larga duración (CLD), parece existir cierta tendencia de convergencia en financiación y provisión siguiendo una misma lógica: desarrollar programas de bienestar social en el marco de una economía de mercado. En concreto, las políticas de CLD se mueven entre dos presiones. La primera es la contención del gasto social público y la segunda es la orientación del gasto hacia actividades de mercado (Matus-López y Rodríguez-Modroño, 2014; Matus-López, 2015; Rodríguez-Modroño y Matus-López, 2016).
En cuanto a las políticas familiares y de cuidados a la infancia (CFI), estas se han visto en parte más protegidas que otras políticas sociales con el enfoque de la inversión social, debido a su carácter instrumental para la mejora del crecimiento y la competitividad, aunque este enfoque también ha contribuido a la reorientación funcional de los Estados de Bienestar (Morel et al., 2012; RodríguezModroño y Matus-López, 2016). Sin embargo, las políticas familiares también se han visto seriamente limitadas con la austeridad, especialmente en los países del Sur de Europa. La austeridad está teniendo impactos devastadores sobre los avances tardíos realizados por estos Estados para cubrir los cuidados a menores y mayores dependientes (León y Pavolini, 2014; Guillén et al., 2016).

De modo que esta nueva era de austeridad representa un desafío clave para el futuro de los Estados de Bienestar que requiere un reconocimiento pleno de la amplitud de sus costes económicos y de su impacto no solo en el corto, sino también en el largo plazo. Este artículo pretende precisamente contribuir a esta discusión de los cambios en los Estados de Bienestar y sus consecuencias futuras intentando responder a las siguientes preguntas de investigación. Si partimos de que estamos en una era de austeridad permanente y en las próximas décadas continuarán las tendencias de los últimos años, tanto económicas como demográficas, ¿cómo se verán afectados los menores y mayores dependientes? Para responder a esta pregunta se efectúa un análisis de la proyección de los gastos en CLD y CFI en el horizonte de 2060 , con objeto de distinguir aquellos países con políticas proinfancia o prodependencia.

La siguiente sección presenta la metodología del estudio, incluyendo el objeto, las fuentes de los datos y la metodología utilizada para las proyecciones. La sección tercera analiza la evolución del gasto público en dependencia e infancia en el periodo 2010-2013 y su evolución con relación al producto interior bruto (PIB). La cuarta sección presenta los principales resultados de la proyección proinfancia y prodependencia en el horizonte de 2060 y la quinta sección concluye con una discusión de las principales implicaciones.

\section{Metodología}

\section{1. Ámbito, definiciones y fuentes de datos}

El ámbito de estudio lo conforman 28 países europeos para los que se dispone de toda la información: Austria, Bélgica, Bulgaria, Croacia, Chipre, República Checa, Dinamarca, Estonia, Finlandia, Francia, Alemania, Grecia, Hungría, Irlanda, Italia, Lituania, Letonia, Luxemburgo, Países Bajos, Noruega, Polonia, Portugal, Rumanía, República Eslovaca, Eslovenia, España, Suecia y Reino Unido.

Los cuidados de larga duración (CLD) comprenden los gastos sociales y sanitarios de servicios para las 
personas con condiciones crónicas y discapacidades que necesitan cuidados permanentes. Tiene dos componentes, uno de salud y otro de servicios sociales. El componente sanitario se obtiene de las cuentas satélites de salud y se refiere a servicios de enfermería y personales (asistencia para las actividades de la vida diaria), y cubre cuidados paliativos y cuidados provistos en instituciones de larga duración o a domicilio. El gasto social incluye el gasto en asistencia para la realización de las actividades instrumentales de la vida diaria.

Los gastos en cuidados a familias e infancia (CFI) se inician entre 1990 y 1995, según el país, y comprenden el apoyo financiero a hogares para el cuidado de hijos u otros familiares y servicios sociales de protección a la familia, especialmente menores. Incluyen tanto prestaciones económicas -asignación por hijo a cargo, prestaciones por nacimiento o adopción, prestaciones por permisos de paternidad, subsidio de natalidad y otras prestaciones en efectivo- como en especie -educación infantil de 0-6 años, alojamiento, asistencia a domicilio y otras prestaciones en especie- (Comisión Europea, 2011).

Las fuentes de información y variables utilizadas fueron las del Fondo Monetario Internacional (FMI) para el PIB 2000-2015, en millones de dólares de 2010, y las proyecciones del PIB 2016-2021 del World Economic Oulook de octubre de 2016. La tasa histórica de crecimiento medio fue calculada sobre el periodo 2000-2015. Los datos de población total, menores de 6 años y de 65 o más años hasta 2013 y las proyecciones de población (Europop2013²) para los tres grupos para 2013-2060 corresponden a Eurostat (2017a), asumiendo el escenario principal.

El gasto público en dependencia o cuidados de larga duración (CLD) se obtuvo de la Unión Europea para 2010 (Lipszyc et al., 2012) y 2013 (Comisión Europea, 2014) y se encuentra expresado en porcentaje del PIB. Las estimaciones de crecimiento del gasto se obtuvieron de las proyecciones en el escenario medio $^{3}$ (AWG reference scenario) y escenario de riesgo4 (AWG risk scenario) para 2013-2060 (Comisión Europea, 2015).

El gasto en políticas familiares se obtuvo de Eurostat (2017b) para el periodo 1990-2014, con distinta longitud de serie según el país en cuestión. Su estimación de escenario medio y de riesgo para 2060 se basa en variaciones de la desviación

${ }_{2}^{2}$ Para las proyecciones de población se utilizó como dato de inicio el 1 de enero de 2013. Las proyecciones se basan en una serie de supuestos sobre la evolución de la fecundidad, mortalidad y migración neta para el periodo 2013-2060.

3 El escenario medio, que combina los escenarios demográficos y de discapacidad constantes, toma el escenario base para las proyecciones de gasto en dependencia: la mitad de las ganancias en esperanza de vida serán sin discapacidad y, por tanto, sin demanda de cuidados.

4 El escenario de riesgo mantiene el supuesto de que la mitad de las ganancias en esperanza de vida no necesitará cuidados, pero añade un escenario de convergencia al alza (hacia la media de la Unión Europea) en los costes unitarios y en la cobertura. estándar histórica de cada serie y país, con una variación negativa de una desviación estándar para el escenario medio y una variación positiva de tres desviaciones estándar para el escenario de riesgo. El criterio 1/3 es discrecional, basado en la evidencia histórica de menor flexibilidad a la baja que al alza.

\subsection{Método}

Se llevaron a cabo dos análisis. El primero estudió la evolución del gasto en CLD y CFI entre 2010-2013. Según los resultados, los países fueron clasificados en un cuadrante de doble entrada: expansión/ recortes y dependencia/infancia, según aumentaron o redujeron los importes de gasto público para cada tipo en el periodo. Se analizaron estas evoluciones en proporción al PIB mediante un análisis de sensibilidad que compara las variaciones de cada tipo de gasto público y las variaciones del PIB para cada país entre 2010 y 2013. Para ello se calculó la elasticidad del gasto en cuidados a la dependencia con respecto al PIB y la elasticidad del gasto en cuidados a la infancia con respecto al PIB. Los resultados permitieron clasificar a los países en un segundo cuadrante de doble entrada: elástico/ inelástico y dependencia/infancia. Los países con valores mayores que 1 muestran una variación porcentual mayor del gasto público en CLD y/o CFI que la variación del PIB. Un valor menor a 1 muestra una variación porcentual menor del gasto público en CLD y/o CFI que la variación del PIB.

En el segundo análisis, se utilizaron las proyecciones de la UE para CLD hasta 2060 en dos escenarios, medio y de riesgo, y se proyectó el gasto en CFI. Este último, al no estar disponible, se estimó para dos escenarios. El primero, denominado "escenario medio", se basa en el supuesto de que el gasto público como porcentaje del PIB varía negativamente una desviación estándar de la evolución histórica de cada país para 2013 y 2060. Esta desviación se calculó sobre el periodo 1994-2014 para 19 países. Las excepciones fueron: Lituania desde 1996, Letonia desde 1997, Estonia y Hungría desde 1999, Chipre, Polonia y Rumanía desde 2000, Bulgaria a partir de 2005 y Croacia a partir de 2008. El segundo escenario, de riesgo, asumió una desviación al alza de hasta tres veces la desviación estándar de cada país hasta 2060 , basada en el mismo periodo histórico.

Se estimaron las dotaciones per cápita de cada gasto y cada escenario, en dólares constantes de 2010. Las cifras per cápita se calcularon sobre el total de la población de 65 o más años de edad para CLD, y sobre menores de 6 años de edad para CFI. Las poblaciones son las estimadas por la Unión Europea para 2060 y las estimaciones de crecimiento del PIB son las del FMI hasta 2021 de cada país y sus respectivas tasas históricas medias para el periodo restante hasta 2060. El indicador utilizado para comparar los resultados fue la relación entre gasto público en CLD per cápita sobre gasto público en 
CFI per cápita. Los resultados se presentaron en dos cuadrantes de doble entrada: relación CLD per cápita/CFI per cápita en 2013 y en 2060. Un cuadrante para el escenario medio y otro para el escenario de riesgo. Estas estimaciones están aún sujetas a modificaciones y nuevas sensibilizaciones.

\section{Gasto en cuidados en un contexto de austeridad y crisis}

No todos los países se han visto igualmente afectados por la crisis. En términos agregados, ocho de los veintiocho países mostraron retrocesos netos en sus economías en 2010-2013. El mayor retroceso se produjo en Grecia, con la crisis económica, política y social más importante de los últimos años en Europa, que le hizo perder un $18 \%$ del PIB. Pero no fue el único. Un segundo grupo, compuesto por Chipre, Portugal y España, mostró retrocesos totales del $8,63 \%, 6,85 \%$ y $5,21 \%$, respectivamente. $Y$ un tercer grupo, con Italia, Croacia y Eslovenia, lo hizo un 3,95\%, 3,5\%, y 3,12 \%, respectivamente. El octavo país, Irlanda, mostró una variación casi neutra; $-0,06 \%$ (Figura 1).

La desaceleración, crisis y, en algunos casos, recesión, presionó a los Gobiernos a realizar ajustes presupuestarios. Las variaciones de los gastos en cuidados fueron en parte una reducción extraordinaria en respuesta al momento económico, pero también forman parte de una política de ajuste de medio y largo plazo que ya se venía produciendo.

Del total de países analizados, nueve mostraron reducciones netas en ambos componentes del gasto público. Como era de esperar, la mayor variación negativa la registró Grecia, con caídas totales de más del $60 \%$ en CLD y cercana al $10 \%$ en CFI, una tendencia que se repite en Eslovenia e Irlanda. No obstante, en los seis restantes (Austria, Bulgaria, Dinamarca, Letonia, Reino Unido y República Checa), se dio una reducción de ambos gastos a pesar del crecimiento de sus economías durante este periodo (Figura 2).

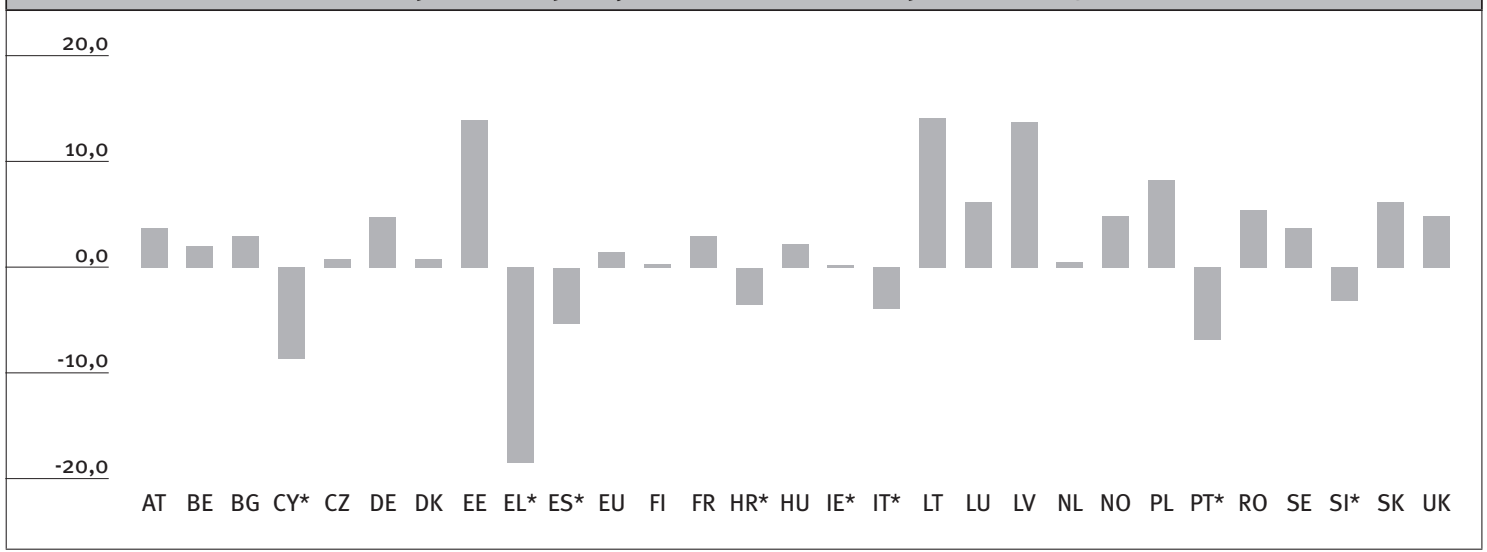

Fuente: Fondo Monetario Internacional (2016).

Figura 2. Variación porcentual del gasto público en cuidados a la infancia y gasto público en cuidados a la dependencia. 28 países europeos y media de la Unión Europea (2010-2013)

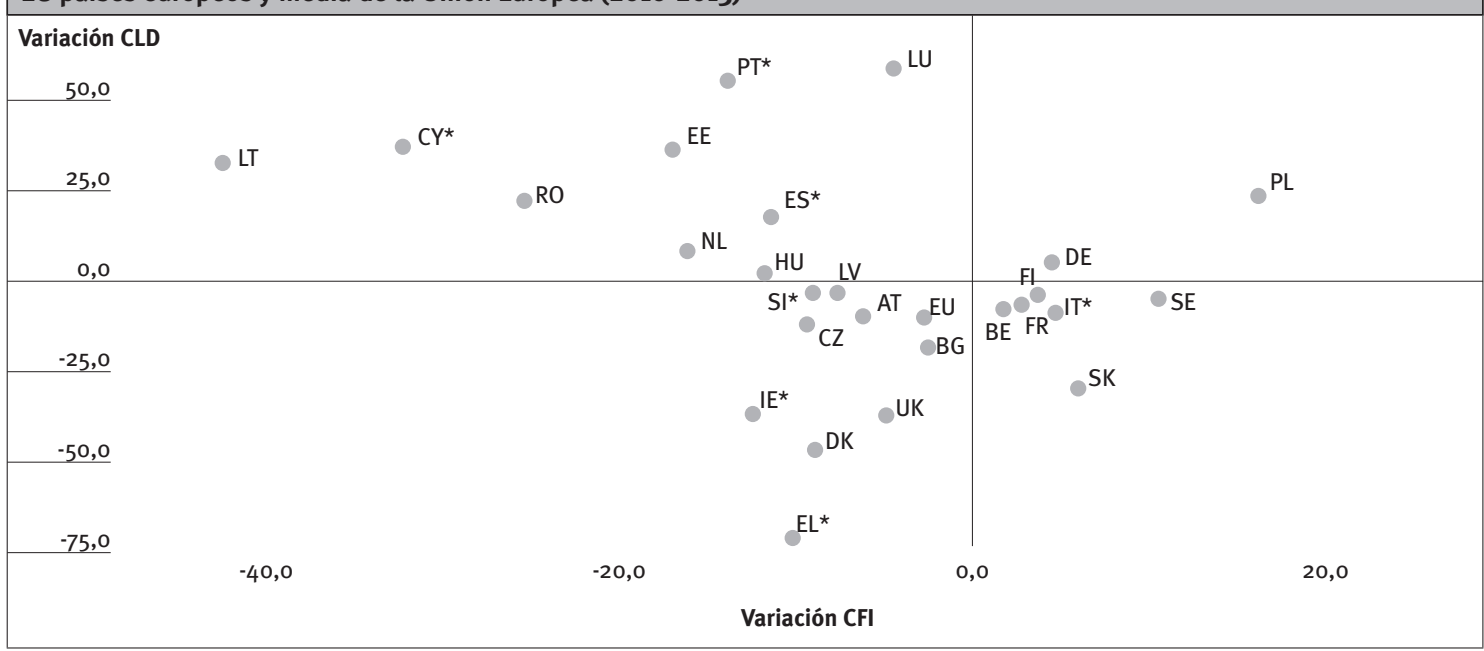

* Países con reducción porcentual del PIB real.

Fuente: Elaboración propia a partir de Lipszyc et al. (2012), Comisión Europea (2015) y Eurostat (2017b). 
Al comparar la evolución del gasto público en CFI y en CLD, se aprecia un mayor ajuste en gastos en infancia que en dependencia. Solo ocho países no redujeron sus gastos en infancia y sus aumentos fueron menores del $15 \%$, con la excepción de Polonia que lo hizo un $16,5 \%$. En cambio, once países aumentaron sus gastos en dependencia y ocho de ellos con incrementos mayores al $15 \%$. Nueve de esos once países se caracterizaron además por compatibilizar una reducción en CFI con un aumento en gasto en CLD. Únicamente Alemania y Polonia aumentaron el gasto público en los dos conceptos.

La preferencia por reducir gasto en CFI más que en CLD se debe en parte a que, en algunos casos, los sistemas de CLD estaban en desarrollo y sus servicios en expansión, por lo que partían de niveles muy bajos y presupuestos más manejables. Los casos más claros son los de España y Portugal, que pese a registrar descensos en la actividad económica y a reducir presupuesto en CFI, aumentaron moderadamente sus dotaciones reales de gasto público en CLD entre 2010 y 2013. Ambos sistemas tenían para entonces poco más de cinco años desde su creación. Algo similar a lo ocurrido con Lituania, Chipre y Rumanía, que sin disponer de un sistema formal de CLD han impulsado los servicios relacionados con la atención a personas dependientes y mayores.
Otra cuestión que es importante considerar es la evolución de los países que hacia 2013 tenían un gasto público en CLD superior al $2 \%$ del PIB: Países Bajos, Finlandia, Francia, Bélgica, Suecia y Dinamarca 5 . En todos ellos existe una tendencia a controlar un gasto que se ha expandido notablemente en las últimas décadas. Así, no es de extrañar que este grupo de países represente una parte importante de aquellos que redujeron el peso de este gasto en el PIB. Solo los Países Bajos registraron una ligera alza y Dinamarca la mayor reducción de todo el grupo (superada únicamente por Grecia).

El peso con relación al PIB se torna, por tanto, relevante. Para ello, se estimaron las elasticidades de ambos gastos públicos con respecto al PIB. Los valores mayores que 1 muestran que el gasto público en CLD o CFI aumentó o se redujo en mayor proporción de lo que lo hizo el PIB. Valores menores que 1 muestran el comportamiento contrario: que los gastos en CLD o CFI fueron menos sensibles a la caída o al alza del PIB.

Los resultados muestran que casi todos los países registraron elasticidades muy cercanas a 1. Es decir, sus variaciones respecto al PIB se ajustan más o menos de forma similar en CLD y CFI (Figura 3).

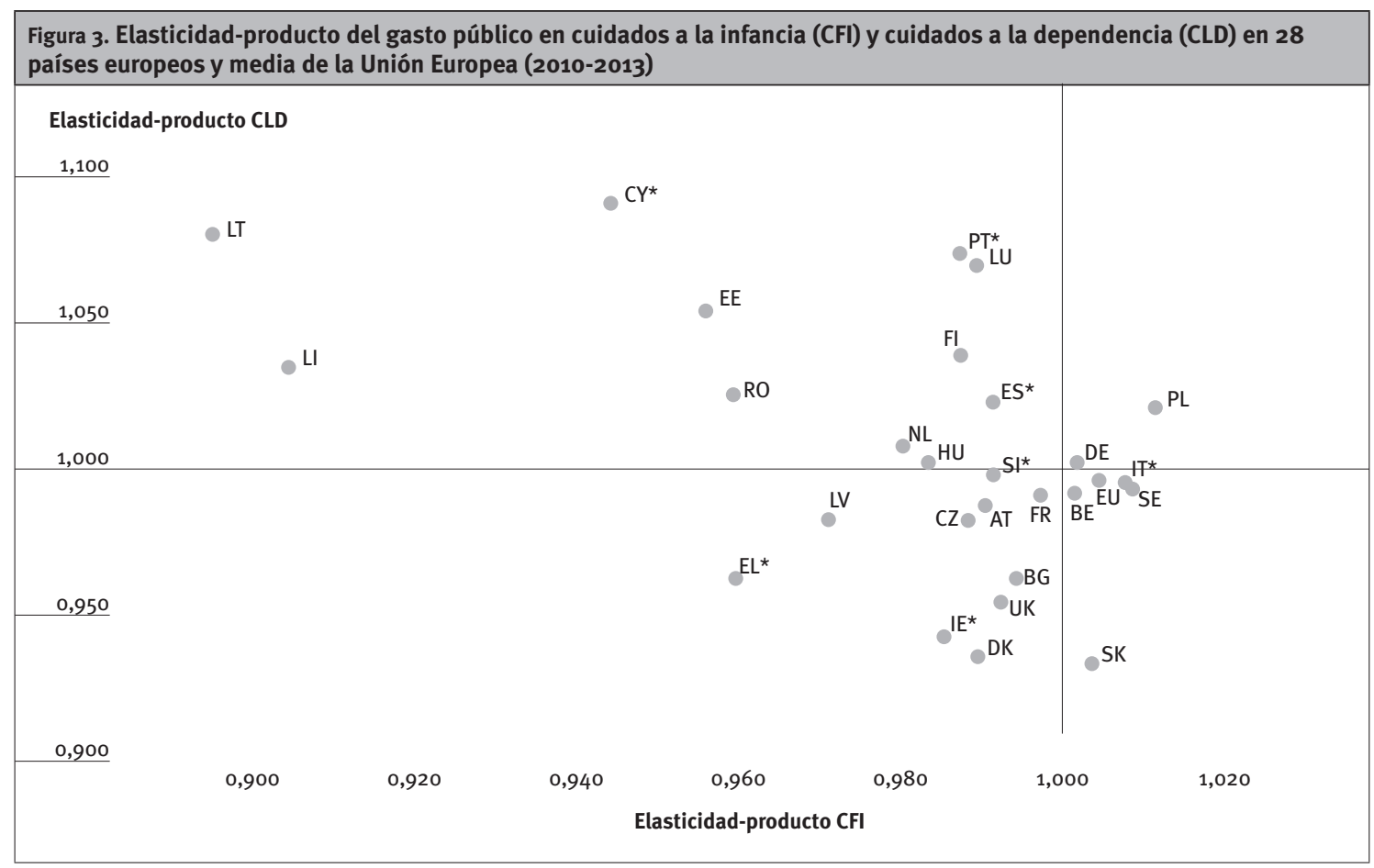

* Países con reducción porcentual del PIB real.

Fuente: Elaboración propia a partir de Lipszyc et al. (2012), Comisión Europea (2015) y Eurostat (2017b). 
En la Figura 3, una nube de puntos compuesta por Lituania, Chipre, Estonia, Rumanía, Portugal y Luxemburgo se aleja del eje central y se dispersa hacia un gasto público más elástico en CLD y menos inelástico en CFI. Es decir, que los gastos de infancia son menos flexibles que los de dependencia, en relación con la variación del PIB. Una posible causa de esta distribución está más bien en la variabilidad de los gastos en CLD, debido a que sus montantes son relativamente pequeños en estos países que, con la excepción de Lituania y Luxemburgo, no superan el $0,6 \%$ del PIB.

\section{Proyecciones de gasto en cuidados a la dependencia y gasto en infancia}

Uno de los problemas de realizar proyecciones es que existen pocos trabajos que propongan evoluciones futuras de gastos, tanto en dependencia como en familia e infancia. Respecto a los CLD, principalmente porque existe escasa información histórica sobre el gasto en este tipo de servicios. Muchos países europeos han implementado estas políticas solo en la última década. Unos las han incorporado en el concepto más amplio de servicios sociales y otros han creado sistemas nuevos de gestión de estos servicios. Asimismo, la definición ha ido ajustándose $\mathrm{y}$, aunque los gastos médicos de cuidados a la dependencia pueden extraerse de las cuentas satélites de salud, la parte de gastos sociales no es igualmente identificable.

En 2012 un documento de trabajo de la Comisión Europea presentó estimaciones de gasto público en CLD para 2010 y una proyección hasta 2060 (Lipszyc et al., 2012). Para ello se basó en las cuentas nacionales satélites de salud y en información solicitada a cada uno de los países. En 2015, esta misma institución presentó nuevas proyecciones en el informe The 2015 Aging Report. Con las mismas fuentes, presentó el gasto público en CLD como porcentaje del PIB en la Unión Europea para 2013 y reajustó las proyecciones hasta 2016.

En el presente artículo se han utilizado los datos de 2010 y 2013 de cada fuente y las proyecciones hasta 2060 del documento de la Unión Europea (Comisión Europea, 2015). En este último trabajo se seleccionaron los dos escenarios promedio: uno combina las evoluciones demográficas, sociales y económicas constantes y el otro supone evoluciones al alza en los determinantes de los costes. Ambos escenarios dan una imagen de mínimos y máximos esperados de la evolución en cada país (Figura 4).

En esta proyección se aprecia una variación limitada en los casos de mayor gasto proporcional al PIB, Países Bajos y Noruega, con valores superiores al $7 \%$, pero con brechas menores a medio punto porcentual. Otros en cambio presentan mayor incertidumbre debido a que el rango de ambas predicciones supera incluso los dos puntos. Este es el caso de Bulgaria, Estonia, Hungría, Letonia, Lituania, República Checa y República Eslovaca. En casi todos los casos, la explicación se encuentra en los bajos niveles de gasto inicial. Las proyecciones sobre montantes menores elevan la dispersión de evoluciones futuras.

Figura 4. Gasto de CLD por países como porcentaje del PIB: 28 países europeos y media de la Unión Europea (máximo en escenario de riesgo para 2060, punto medio en escenario medio 2060 y mínimo para 2013)

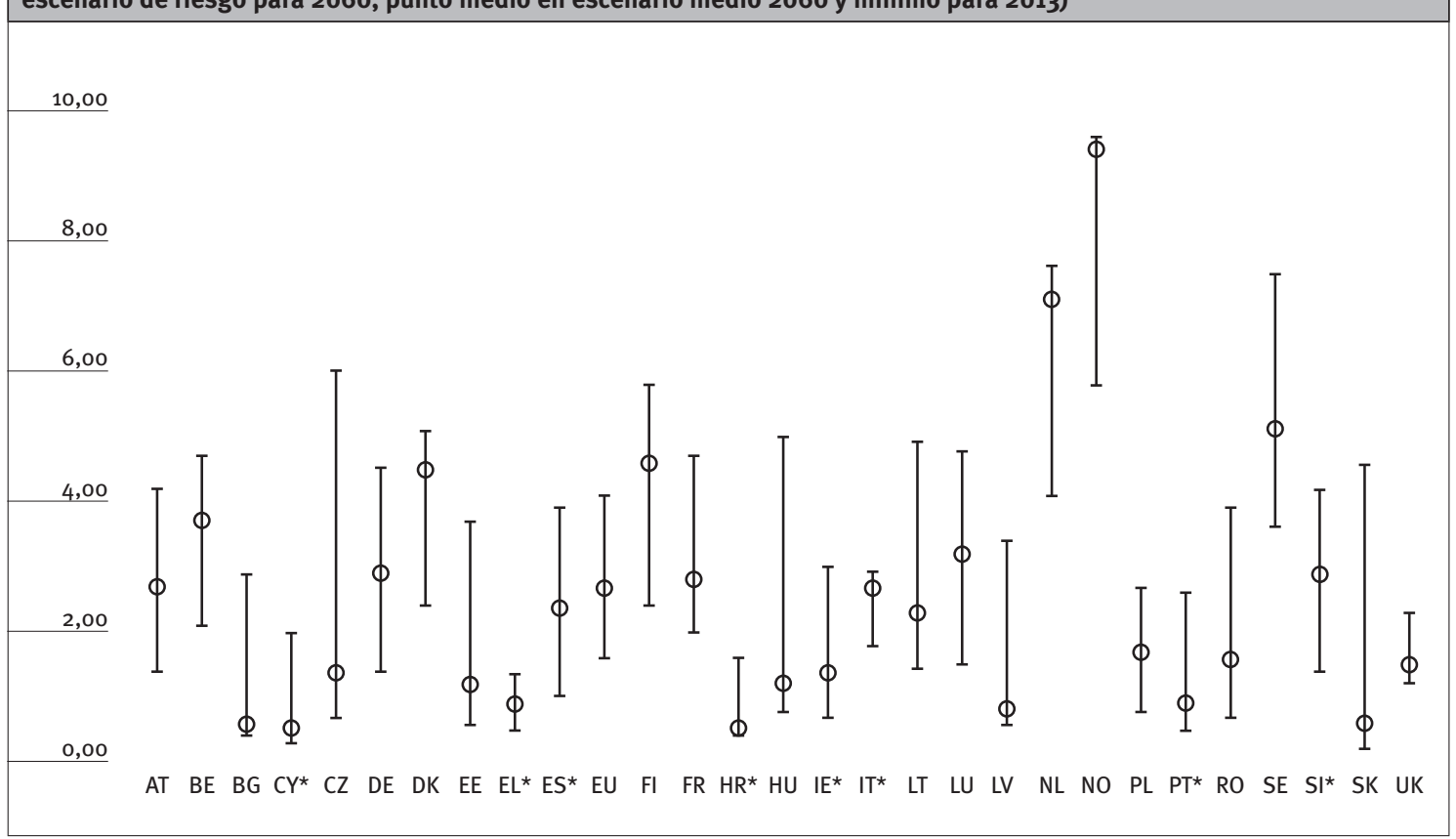

* Países con reducción porcentual del PIB real.

Fuente: Elaboración propia a partir de Comisión Europea (2015). 
Como ya se ha comentado en la metodología, la proyección para CFI se realizó teniendo en cuenta su evolución desde 1994 hasta 2014. Por lo tanto, dependen de la variabilidad histórica de cada gasto público en cada país. Las variaciones son menores, ya que la variabilidad entre países también es menor. Por ejemplo, la desviación estándar de gasto público en CLD en 2013 fue de 87,5\% de la media. En CFI para el mismo año, fue del $41,9 \%$ de la media (Figura 5).

Pese a que las variaciones entre uno y otro escenario son menores a medio punto porcentual en la mayoría de los países europeos, se pueden identificar algunos con mayor incertidumbre que otros. La conjugación de estos y los de las proyecciones de CLD hacen tomar con precaución los resultados para Bulgaria, Lituania, Estonia y Eslovaquia.
Las siguientes figuras representan la relación entre gasto público en CLD per cápita sobre gasto público en CFI per cápita (en dólares constantes de 2010) en 2013 y en 2060 para el escenario medio y para el escenario de riesgo. En las dos figuras, un amplio número de países se sitúa por debajo del 1 en su relación de gastos per cápita en CLD y CFI, es decir tanto en 2013 como en 2060 su gasto per cápita en políticas de apoyo a la familia y cuidado de menores es superior al gasto per cápita en cuidado de dependientes, aunque se observa en el futuro un mayor peso del gasto per cápita en dependencia.

En el caso del escenario medio (Figura 6), la mayoría de países se ubican por debajo de la línea de 45 grados. Esta línea muestra una evolución neutra. Es decir, los puntos situados en la línea representan

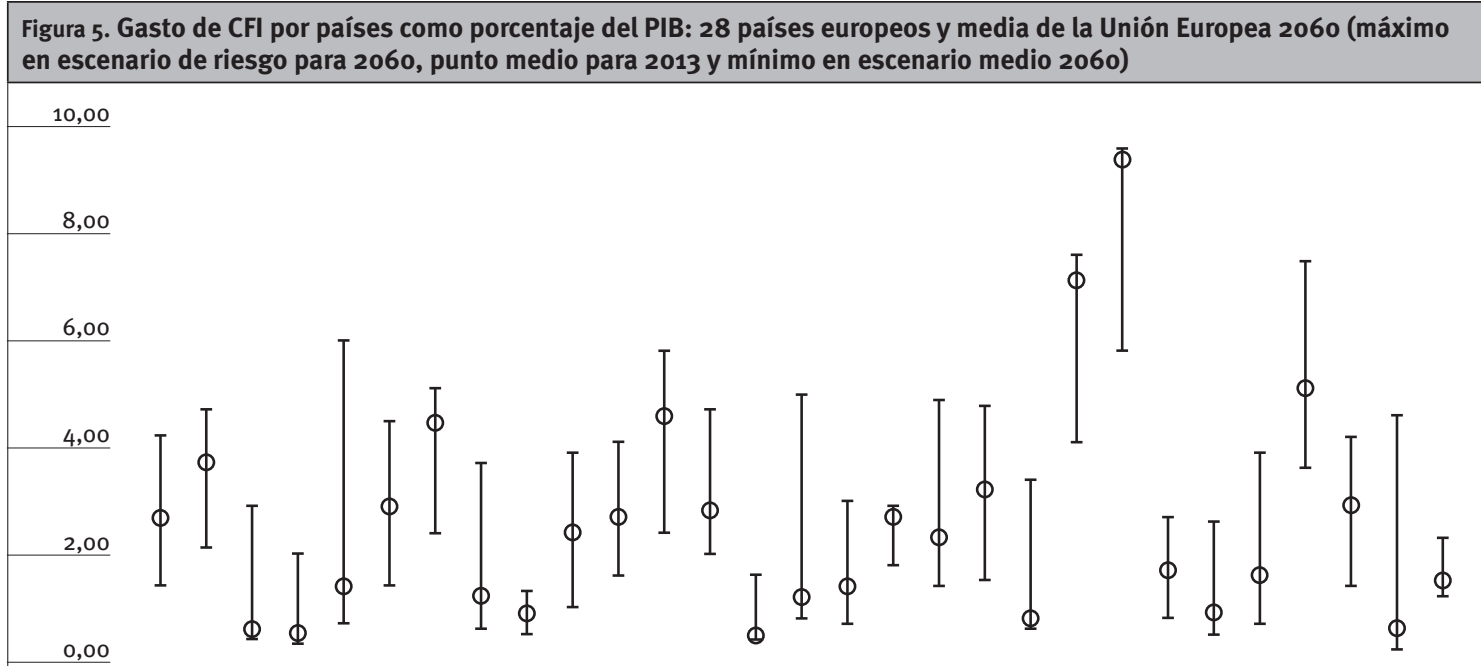
AT BE $\quad B G \quad C Y^{\star} C Z$ DE DK EE EL* ES* EU FI $\quad F R$ HR* HU IE* IT* LT LU LV NL NO PL PT* RO SE SI* SK UK

* Países con reducción porcentual del PIB real.

Fuente: Elaboración propia a partir de Eurostat (2017a, 2017b).

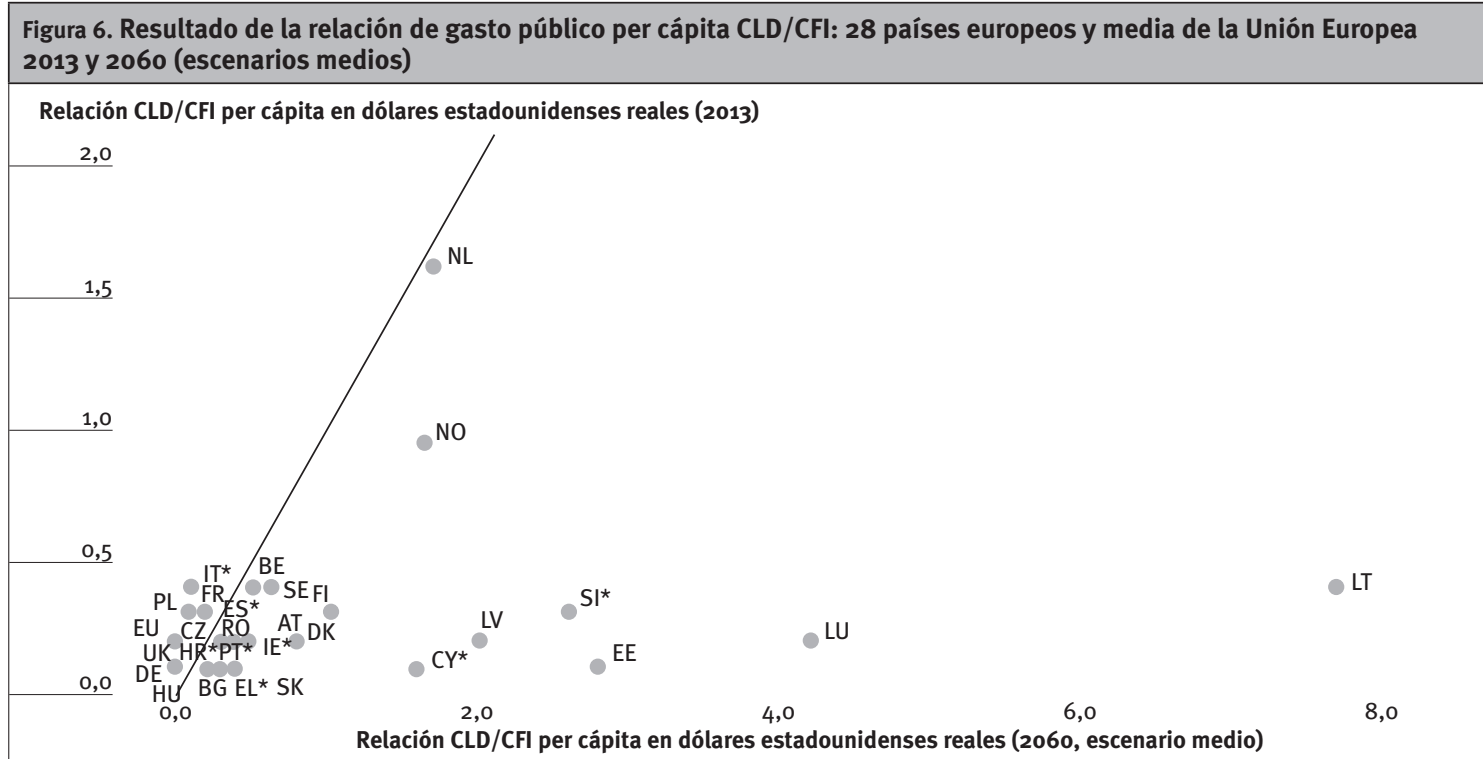

Fuente: Elaboración propia. 
países en los que el gasto per cápita en dependencia sigue manteniendo la misma proporción del gasto per cápita en infancia en 2060 que en 2013. LoS puntos situados bajo la línea de 45 grados son países en los que el gasto per cápita en dependencia aumenta más que el gasto per cápita en infancia. Casi la totalidad de los países muestra un desplazamiento temporal a favor del gasto per cápita en dependencia que en infancia en el horizonte 2060.

Solo ocho países se encuentran a la izquierda de la línea de 45 grados (Alemania, Bélgica, España, Francia, Italia, Países Bajos, Polonia y Reino Unido), es decir, son los únicos que presentan una tendencia más proinfancia que prodependencia. Sin embargo, su peso en el total europeo conduce a que la media de la Unión Europea se encuentre también sobre la línea divisoria.

La nube de puntos que más se aleja hacia una tendencia prodependencia está compuesta en parte por aquellos países sobre los que se debe tener precaución por la variabilidad de sus indicadores, como Lituania y Estonia, o por países con un gasto todavía escaso en dependencia en 2013, lo cual explicaría este comportamiento.

El caso es distinto para los otros dos puntos alejados: Países Bajos y Noruega. Ambos parten con modelos consolidados de cuidados a la infancia y a la dependencia y muestran proyecciones más o menos estables a futuro. En ambos casos los gastos en dependencia en 2013 eran, proporcionalmente al gasto en infancia, de los más elevados de Europa. Así, en el escenario medio de 2060, se mantienen entre los de mayor gasto Países Bajos, con proporciones similares entre ambos tipos de gasto per cápita, y Noruega, con una leve inclinación hacia un aumento de los gastos per cápita en dependencia.
En el escenario de riesgo (Figura 7) las tendencias generales son similares. Una vez más un grupo de países, principalmente de Europa del Este, muestra un desplazamiento hacia indicadores prodependencia. Una tendencia que hay que tomar con cautela por los determinantes señalados de variabilidad y de bajo gasto inicial. Noruega y Países Bajos vuelven a situarse por encima del grupo de países, pero cercanos a la línea de 45 grados.

Para poder comparar mejor ambos escenarios es conveniente ampliar los dos últimos gráficos sobre la línea de puntos central, limitando las razones a 1. En este ejercicio (Figura 8) se pueden apreciar cinco cuestiones. En primer lugar, la mayoría de los países europeos tienen razones inferiores a 1 (20 países en el escenario medio y 19 en el escenario de riesgo), esto es, tienen y seguirán teniendo en $\mathbf{2 0 6 0}$ un gasto per cápita en dependencia inferior al gasto per cápita en políticas familiares. A pesar de que los países europeos aumentan su gasto en dependencia, estos incrementos no compensan suficientemente el envejecimiento de la población. En segundo lugar, dos terceras partes de los países europeos se encuentran en este rango de valores; es decir, la variación entre los países europeos es escasa. En tercer lugar, existe una mayoría en ambos escenarios que muestra una tendencia prodependencia. En cuarto lugar, el escenario de riesgo y el escenario medio no muestran grandes diferencias agregadas. El número de países por debajo y por encima de la línea de 45 grados se mantiene. En quinto lugar, los países con mayor peso poblacional y económico de Europa muestran una tendencia proinfancia: Alemania, Reino Unido, Francia, Italia y España, además de Polonia y la media de la Unión Europea. Es decir, el aumento del gasto público en dependencia en proporción a las personas de 650 más años de edad en 2060 es menor que el aumento del gasto en infancia con respecto a la población menor de seis años en 2060.

Figura 7. Resultado de la relación de gasto público per cápita CLD/CFI: 28 países europeos y media de la Unión Europea 2013 y 2060 (escenario de riesgo)

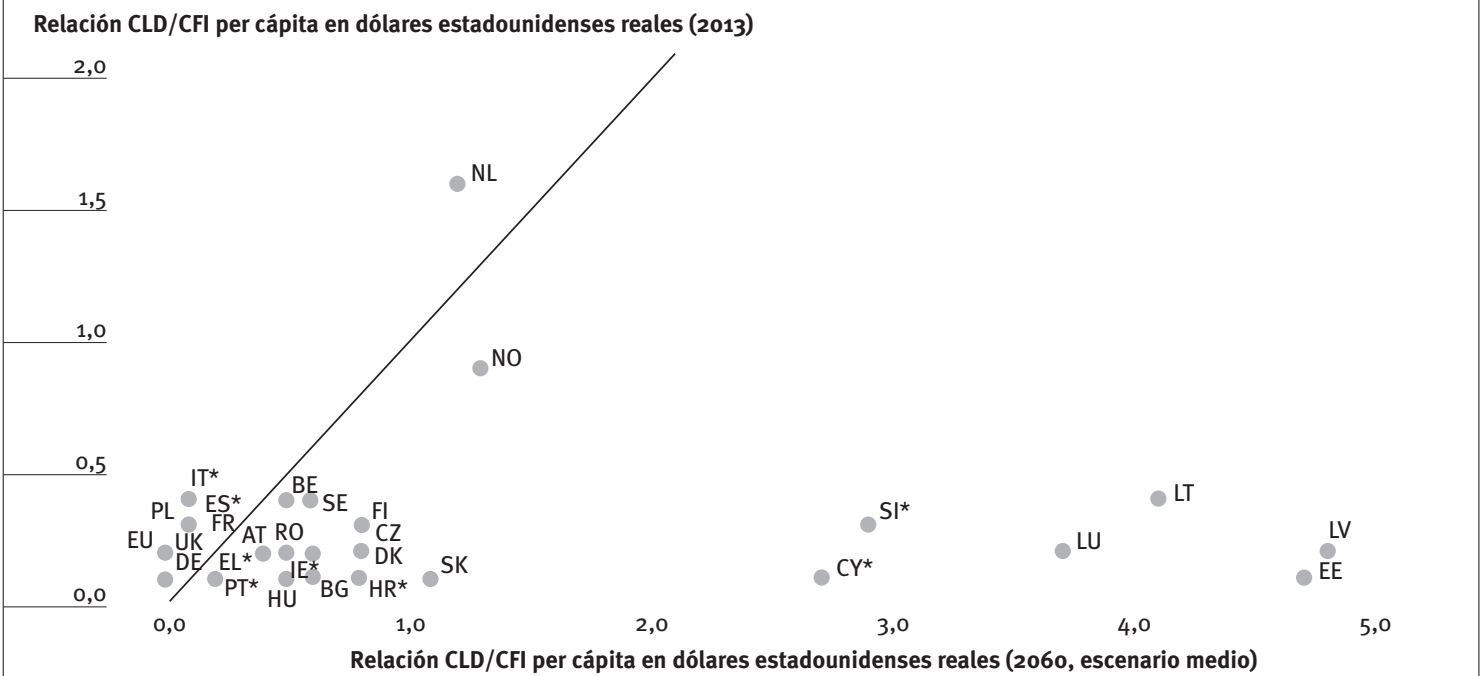

Fuente: Elaboración propia. 
Relación CLD/CFI per cápita en dólares estadounidenses reales (2013)

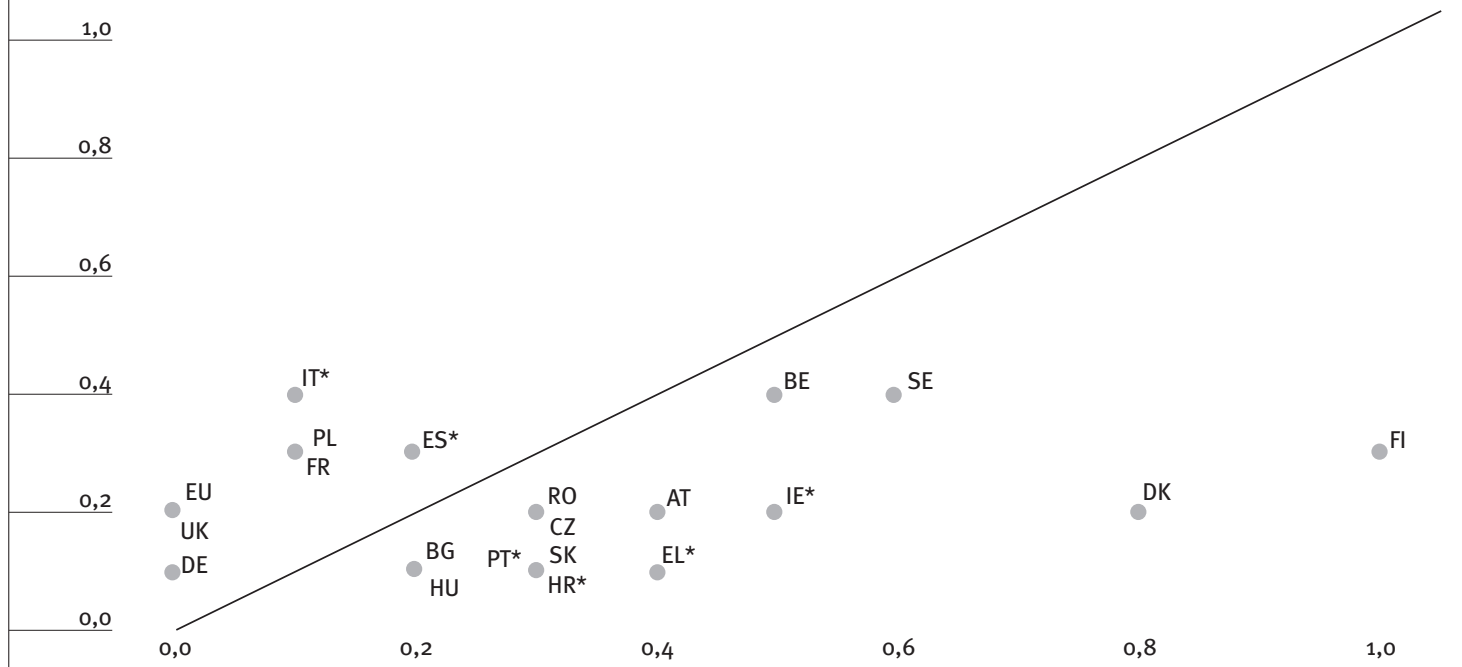

Relación CLD/CFI per cápita en dólares estadounidenses reales (2060, escenario medio)

Relación CLD/CFI per cápita en dólares estadounidenses reales (2013)

1,0

0,8

0,4

0,2

0,0

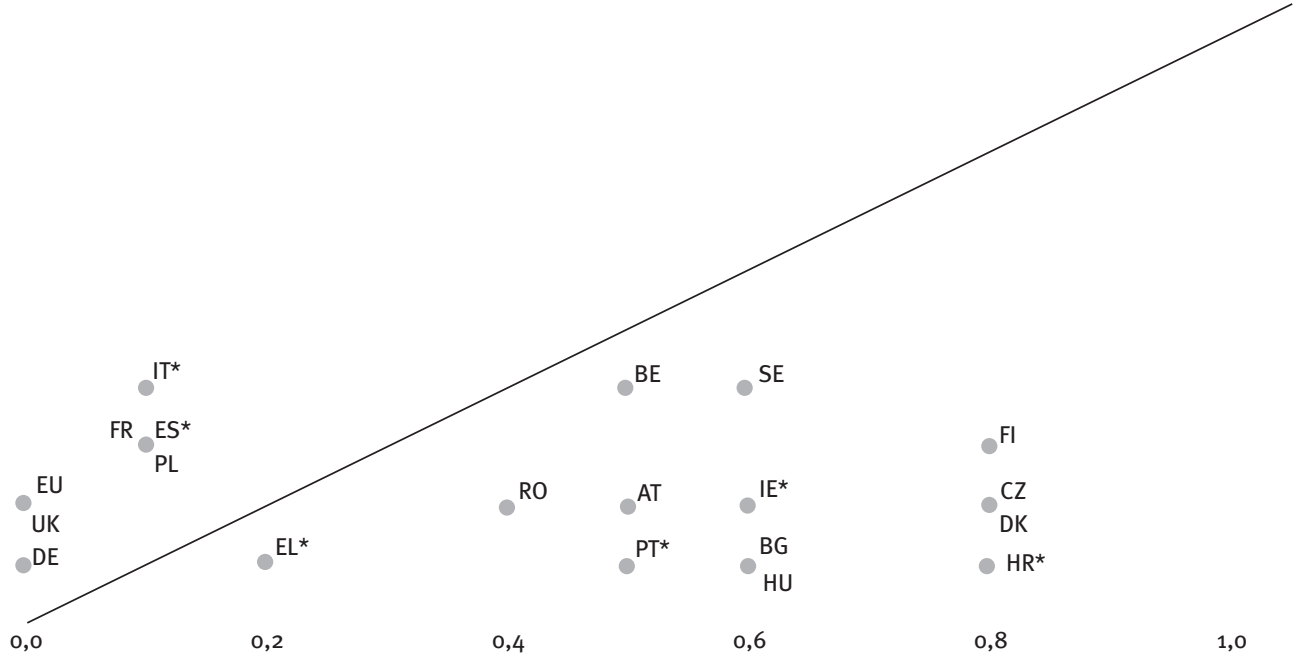

Relación CLD/CFI per cápita en dólares estadounidenses reales (2060, escenario riesgo)

Fuente: Elaboración propia.

Por último, los países con modelos de cuidados a la dependencia más consolidados y con mayor presupuesto en 2013 muestran una tendencia prodependencia en el escenario medio 2060: Dinamarca, Finlandia, Suecia y Bélgica. Si se analiza el escenario de riesgo, siguen una tendencia prodependencia países que hoy tienen los gastos públicos más bajos respecto a Europa: República Checa, Croacia, Bulgaria, Rumanía, Hungría y Portugal. A estos últimos se une Austria, que parece ser más sensible al escenario de riesgo.

\section{Discusión y conclusiones}

Desde principios de los noventa la lógica neoliberal ha guiado las reformas en las políticas sociales y de cuidados de los países europeos, orientándolas hacia un fortalecimiento de los principios de eficiencia económica en la provisión de los cuidados. Las reformas se han llevado a cabo buscando un equilibrio entre dos fuerzas, la ampliación de la cobertura de los servicios de acuerdo a las crecientes necesidades sociales de cuidados y la contención del 
gasto público (Rodríguez-Modroño y Matus-López, 2016).

La crisis económica mundial de 2008 ha servido para agudizar las presiones sobre la contención del gasto público. Como expresan Farnsworth e Irving (2015), la austeridad se ha convertido en la lógica organizadora del gasto público, sobre todo del gasto social. La mayoría de los países europeos han reducido o contenido sus gastos sociales, incluyendo aquellos destinados a las políticas de cuidados. De los veintiocho países analizados, solo Alemania y Polonia aumentaron sus gastos tanto en dependencia como en políticas familiares y de cuidados a la infancia durante el periodo 2010-2013. El resto redujo en uno $u$ otro sentido sus gastos y diez países mostraron reducciones netas en ambos componentes del gasto público. Destacan los ajustes realizados en ambos gastos en los países europeos sometidos a vigilancia, como Grecia o Irlanda. Pero no solo en los países en recesión se han impuesto las políticas de austeridad. En siete países europeos se redujeron ambos gastos pese a que sus economías crecieron, evidenciando que el discurso de la austeridad domina las políticas sociales de todos los países, incluso de aquellos sin recesión o con esta ya finalizada. El mayor ajuste se ha dado en los gastos en infancia, debido a que muchos de los sistemas de atención a la dependencia estaban iniciando su desarrollo, como los de países del Sur de Europa o del Este, por lo que partían de niveles muy bajos y presupuestos más manejables. Por el contrario, aquellos países europeos que partían de mayores gastos en dependencia con relación a su PIB han intentado controlarlos.

La proyección de los gastos en dependencia e infancia per cápita para 2060 y la relación entre ambos muestran para la mayoría de países europeos un desplazamiento a favor del gasto per cápita en dependencia. Incluso los países con modelos de cuidados a la dependencia más consolidados y con mayor presupuesto en 2013 (Dinamarca, Finlandia, Suecia y Bélgica) siguen mostrando una tendencia prodependencia en el escenario medio para 2060. Solo los países con mayor peso poblacional y económico de Europa muestran una tendencia proinfancia en los próximos años. Sin embargo, los incrementos en el gasto para la dependencia no son suficientes para compensar el creciente envejecimiento de la población europea. Mientras que la población menor de 6 años en la
Unión Europea de los 28 se reducirá un $4 \%$ en el periodo 2013-2060, la población mayor de 65 años aumentará un $60 \%$. Aunque el gasto en dependencia se incrementa en los próximos años, normalmente más que el gasto en infancia, el esfuerzo es mínimo en comparación con las necesidades del cambio demográfico, de manera que el gasto per cápita en políticas de apoyo a la familia y cuidado de menores seguirá siendo superior al gasto per cápita en cuidado de dependientes en 2060.

El análisis efectuado muestra, por tanto, un crecimiento en los gastos de provisión pública de cuidados de las próximas cinco décadas insuficiente con respecto al incremento de la demanda de cuidados. Este déficit de cuidados formales conlleva, sobre todo en una época como la actual de bajos salarios y precarización de las relaciones laborales, que los cuidados deban ser proporcionados por los hogares a través de trabajo informal, remunerado o no remunerado, incrementando las desigualdades tanto de clase y de etnia como de género. Los hogares se ven y verán forzados a emplear, muchas veces sin contrato, a trabajadores de forma precaria y a bajo coste $o$ a proveer estos cuidados por miembros de la familia, normalmente mujeres. Esta refamilización en la provisión social de los cuidados tiene repercusiones negativas cruciales sobre la participación laboral de las mujeres de forma estable y a tiempo completo, la fecundidad, o el mismo desarrollo de los países. La participación laboral de las mujeres se ve seriamente afectada tanto por el cuidado de los hijos (Thévenon, 2009; Hegewisch y Gornick, 2011; Boeckmann et al., 2015), como por el cuidado a mayores dependientes (Kotsadam, 2011), especialmente cuando no existe una provisión pública adecuada o asequible (Saraceno, 2010), los hogares no disponen de recursos financieros suficientes (Sarasa y Billingsley, 2008), o se requieren cuidados intensivos (Crespo, 2006). Esta situación afecta a todos los países europeos, pero especialmente a los del sur de Europa, donde un importante porcentaje de mujeres proporciona cuidados informales intensivos a sus familiares mayores (Naldini et al., 2016). De esta manera, en la medida en que se ofertan menos servicios públicos de cuidados a menores, mayores o dependientes que los demandados por la sociedad, se transfiere la responsabilidad y carga de trabajo de cuidados a las familias, limitando la autonomía de las mujeres para desarrollar tanto su carrera profesional como, en general, su vida. 
BOECKMANN, I.; MISRA, J.; y BUDIG, M. (2015): “Cultural and institutional factors shaping mothers' employment and working hours in postindustrial countries", Social Forces, vol. 93, núm. 4, págs. 1301-1333.

COMISIÓN EUROPEA (2011): ESSPROS Manual. The European System of integrated Social PROtection Statistics (ESSPROS) [/http://ec.europa.eu/ eurostat/documents/3859598/5917481/KSRA-11-014-EN.PDF/ee86d517-3348-4C20-94eea37c330755b1)].

- (2014): “The 2015 Ageing Report: Underlying Assumptions and Projection Methodologies", European Economy 8-2014, Economic and Financial Affairs.

- (2015): "The 2015 Ageing Report: Economic and budgetary projections for the 28 EU Member States (20132060)". European Economy 3-2015. Economic and Financial Affairs.

CRESPO, L. (2006): “Caring for Parents and Employment Status of European Mid-Life Women", Working Paper No. 0615, Centro de Estudios Monetarios y Financieros.

EUROSTAT (2017a): Population data, Population projections data [«http://ec.europa.eu/eurostat/web/ population-demography-migration-projections/ population-data/main-tables〉].

- (2017b): Social protection data [<http://ec.europa. eu/eurostat/web/social-protection/data/ database $)$.

FARNSWORTH, K.; y IRVING, Z. (eds.) (2011): Social Policy in Challenging Times: Economic Crisis and Welfare Systems, Bristol, Bristol University Press.

- (2015): Social Policy in Times of Austerity. Global Economic Crisis and the New Politics of Welfare, Bristol, Policy Press.
FERRERA, M.; HEMERIJCK, A.; y RHODES, M. (2000): The Future of Social Europe: Recasting Work and Welfare in the New Economy, Oeiras, Celta Editora.

FONDO MONETARIO INTERNACIONAL (2016): World Economic Outlook [rhttp://www.imf.org/external/pubs/ $\mathrm{ft} / w e o / 2016 / 02 / \nu]$.

GUILLÉN, A. M.; GONZÁLEZ-BEGEGA, S.; y LUQUE BALBONA, D. (2016): "Austeridad y ajustes sociales en el Sur de Europa. La fragmentación del modelo de bienestar Mediterráneo", Revista Española de Sociología, vol. 25, núm. 2, pp. 261-272.

HEGEWISCH, A.; y GORNICK, J. C. (2011): "The impact of work-family policies on women's employment: a review of research from OECD countries", Community, Work \& Family, vol. 14, núm. 2, pp. 119-138.

HEMERIJCK, A. (2012): Changing Welfare States, Oxford, Oxford University Press.

JESSOP, B. (2015): “Neoliberalism, finance-dominated accumulation and enduring austerity: a cultural political economy perspective", en FARNSWORTH, K., IRVING, Z. (eds.), Social Policy in Times of Austerity. Global Economic Crisis and the new Politics of Welfare, Bristol, Policy Press, pp. 87-112.

KARGER, H. (2014): “The Bitter Pill: Austerity, Debt, and the Attack on Europe's Welfare States", The Journal of Sociology \& Social Welfare, vol. 41, núm. 2, págs. 33-54.

KOTSADAM, A. (2011): “Does informal eldercare impede women's employment? The case of European Welfare States", Feminist Economics, vol. 17, núm 2, pp. 121-144.

LEÓN, M.; y PAVOLINI, E. (2014): “Social investment or back to Familism: the impact of the economic crisis 
on family and care policies in Italy and Spain", South European Society and Politics, vol. 19, núm. 3, pp. 353-369.

LIPSZYC, B.; SAIL, E.; y XAVIER, A. (2012): “Long-term care: need, use and expenditure in the EU27", European Economy Economic Papers 469, Economic and Financial Affairs [<http:// ec.europa.eu/economy_finance/publications/ economic_paper/2012/pdf/ecp469_en.pdf〉].

MATSAGANIS, M. (2012): "Social policy in hard times: The case of Greece”, Critical Social Policy, vol. 32, núm. 3, pp. 406-421.

MATUS-LÓPEZ, M. (2015): “Tendencias en las políticas de atención a la dependencia de ancianos y sus reformas", Cadernos de Saúde Pública, vol. 31, núm. 12, pp. 2475-2481.

MATUS-LÓPEZ, M.; y RODRÍGUEZ-MODROÑO, P. (2014): "Presiones de oferta y demanda sobre políticas formales de cuidados en Latinoamérica", Revista del CLAD Reforma y Democracia, núm. 6o, pp. 103-130.

MOREL, N.; PALIER, B.; y PALME, J. (eds.) (2012): Towards a Social Investment Welfare State? Ideas, Policies and Challenges, Bristol, Policy Press.

NALDINI, M.; PAVOLINI, E.; y SOLERA, C. (2016): "Female employment and elderly care: the role of care policies and culture in 21 European countries", Work, Employment and Society, vol. 30, núm. 4, pp. 607-630.

OCDE (2016): Health at a Glance 2016, Paris, Organization for Economic Cooperation and Development.

PALIER, B. (2010): A Long Goodbye to Bismarck? The Politics of Welfare Reform in Continental Europe, Amsterdam, Amsterdam University Press.
PAVOLINI, E.; LEÓN, M.; GUILLÉN, A. M.; y ASCOLI, U. (2015): "From austerity to permanent strain? The EU and welfare state reform in Italy and Spain", Comparative European Politics, vol. 13, núm. 1, pp. 56-76.

PIERSON, P. (2002): “Coping with permanent austerity: welfare state restructuring in affluent democracies", Revue Française de Sociologie, vol. 43, núm. 2, pp. 369-406.

RODRÍGUEZ-MODROÑO, P.; y MATUS-LÓPEZ, M. (2016): "Políticas de cuidados a la infancia y mayores dependientes. Evolución y condicionamiento pro-mercado", Revista del CLAD Reforma y Democracia, núm. 66, pp. 99-130.

ROMANO, S.; y PUNZIANO, G. (2015): "Introduction: The Remains of the European Social Contract”, The European Social Model Adrift. Europe, Social Cohesion and the Economic Crisis, Farnham, Ashgate, pp. 1-15.

SARACENO, C. (2010): "Social inequalities in facing old-age dependency: a bi-generational perspective", Journal of European Social Policy, vol. 20, núm. 1, pp. 32-44.

SARASA, S.; y BILLINGSLEY, S. (2008): "Personal and household caregiving from adult children to parents and social stratification", en SARACENO, C. (ed.), Families, Ageing and Social Policy: Intergenerational Solidarity in European Welfare States, Cheltenham, Edward Elgar, pp. 123-146.

TAYLOR-GOOBY, P. (2002): "The Silver Age of the Welfare State. Perspectives on Resilience", Journal of Social Policy, vol. 31, núm. 4, pp. 597-621.

THÉVENON, O. (2009): “Increased Women's Labour Force Participation in Europe: Progress in the WorkLife Balance or Polarization of Behaviours?", Population, vol. 64, núm. 2, pp. 235-272. 